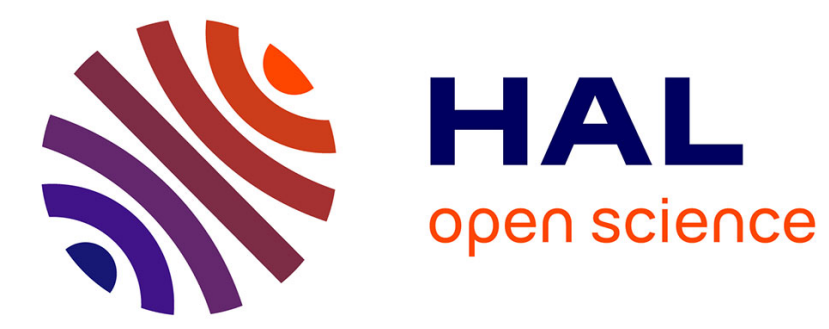

\title{
Gros mots et insultes des adolescents
}

Claudine Moïse

\section{To cite this version:}

Claudine Moïse. Gros mots et insultes des adolescents. Revue de l'enfance et de l'adolescence RAFEFGRAPE, 2011, Sales et méchants, 82, pp.29-37. hal-02468816

\section{HAL Id: hal-02468816 https://hal.science/hal-02468816}

Submitted on 6 Feb 2020

HAL is a multi-disciplinary open access archive for the deposit and dissemination of scientific research documents, whether they are published or not. The documents may come from teaching and research institutions in France or abroad, or from public or private research centers.
L'archive ouverte pluridisciplinaire HAL, est destinée au dépôt et à la diffusion de documents scientifiques de niveau recherche, publiés ou non, émanant des établissements d'enseignement et de recherche français ou étrangers, des laboratoires publics ou privés. 


\section{Gros mots et insultes des adolescents}

Claudine Moïse

(2011) Moïse C., « Gros mots et insultes des adolescents », Sales et méchants, Revue GRAPE, Lettre de l'enfance et de l'adolescence $\mathrm{n}^{\mathrm{o}} 82$, pages $29-37$

\section{Résumé}

Entre gros mots et insultes des adolescents, les adultes ont bien du mal à se repérer et à ne pas se sentir menacés. Cet article s'attache à montrer, à travers la distinction linguistique de ces faits de langue, que les pratiques adolescentes, loin d'être nouvelles, rendent compte d'évolutions et d'usages propres aux langues et aux normes sociales. Il s'agit alors de repérer les activités ludiques et les jeux interactionnels typiques de cet âge-là, sans négliger certaines provocations agressives qui peuvent exprimer de réelles souffrances intimes.

\section{Mots-clés}

Actes menaçants, gros mot, juron, insulte, expressivité, interaction, sexualité.

Les adolescents, dans la période intense de changement et de construction de soi qu'ils traversent, cherchent par des processus d'identification qui leur sont propres, codes vestimentaires et réseaux sociaux par exemple, à se reconnaître les uns les autres tout en se distinguant des adultes. Les pratiques langagières font aussi partie des ressources à la disposition des adolescents pour signifier connivence, singularité et identité. Je voudrais montrer ici combien certains phénomènes linguistiques utilisés par des adolescents, les gros mots, jurons et insultes, notamment à connotation sexuelle, participent de façon inévitable et nécessaire à l'expression de soi, que l'on soit dans des usages ludiques ou plus agressifs ${ }^{1}$. Il s'agira de voir aussi que le passage des jurons ou insultes à une violence verbale ${ }^{2}$ plus caractérisée traduit aussi des mal-être qui dépassent la simple mise en scène de soi adolescente.

\footnotetext{
${ }^{1}$ Ma réflexion ici s'appuie sur des travaux de collègues sociolinguistes et sur toutes les recherches que je mène depuis quelques années autour de la violence verbale à partir d'enquêtes ethnographiques, et plus récemment sur les parlers adolescents J'ai suivi aussi des mineurs, auteurs et victimes de violences sexuelles, dans le cadre de thérapies sexuelles.

2 Voir les travaux du groupe de recherche sur la violence verbale dirigés par Claudine Moïse (www.violenceverbale.fr). «La violence verbale fulgurante est une montée en tension contextualisée qui se décline à travers différentes étapes (incompréhension, négociation, évitement, renchérissement, renforcement...) marquées par des déclencheurs de conflit (matériels ou symboliques), des marqueurs discursifs de rupture (durcisseurs, mots du discours, effets syntaxiques) et des actes de langage
} 


\section{Quelques préalables}

\section{La force tabou de la langue}

La langue peut servir une volonté de transgression, et le sentiment de vulgarité, d'agressivité ou de grossièreté repose souvent sur des formes choquantes et décalées par rapport aux codes sociaux attendus. Cet usage tabou du langage s'actualise dans trois domaines sémantiques, le sacré ${ }^{3}$ (la religion), les excréments (la scatologie) et la sexualité. Ils sont condamnés parce qu'à travers leur usage se manifestent les interdits d'une société.

Dans la société française d'aujourd'hui les mots transgressifs les plus utilisés sont ceux liés à la sexualité. Quand on parle d'elle, la sexualité, d'une façon ou d'une autre, se dit à travers des mots mis à distance. Soit, ils font partie d'un registre perçu comme spécialisé, «la vulve » (qui apparaît rarement dans le dictionnaire mais se voit suppléé par « sexe de la femme ») ou « le pénis » par exemple. Soit, dans l'usage courant, on leur préfère des hypocoristiques, «la foufoune », « le zizi ». Mais plus encore, les termes de la sexualité, mots tabou ${ }^{4}$, reposent bien souvent sur des formes, ressenties comme grossières et crues, voire « sales », qui servent à transgresser l'interdit sexuel ; ils se font alors « gros mots » de ceux qui, contraires aux bienséances, s'encanaillent et offensent la pudeur. S'ils concernent la femme et le féminin, bien souvent sujets de déconsidération dans la langue (Duchêne et Moïse 2010), ils sont d'autant plus vulgaires. Par exemple, le terme si banal de «con » est révélateur du dénigrement à l'égard de la femme et du peu de considération de sa sexualité. Apparu au $12^{\mathrm{e}}$ siècle du latin connus, il fait référence à la bêtise («parler, raisonner comme un con») et à cette femme qui déraisonne sous l'effet de son utérus (qui renvoie à l'hystérie). De même que "putain » ou «salope » (contrairement à « salaud ») sont associés à une femme à la sexualité considérée comme « dépravée ».

\section{Des gros mots aux insultes}

dépréciatifs directs (harcèlement, mépris, provocation, déni, insulte...) à visée de domination » (Moïse et Schultz-Romain à paraître).

${ }^{3}$ La société québécoise, fortement imprégnée jusqu'aux années 1960 de valeurs catholiques (Vincent 1984) a gardé beaucoup de jurons, des sacres, liés à la religion et autres que ceux rattachés à «Dieu » («Tabarnak! », «Viarge ! », « Câlisse ! »), contrairement à la société française (« Nom de Dieu ! ).

${ }^{4}$ Voir sur la langue tabou les écrits de Delvau [1900], 1990, Rouyarenc (1996), Huston (2002), 
Les mots tabou peuvent être à la fois mot grossier, juron ou insulte. S'il a valeur de transgression langagière, le mot «merde » par exemple n'occupe pas toujours la même fonction pragmatique (Huston 2002: 20). Le gros mot repose sur la fonction référentielle du langage et en appelle donc à l'objet désigné («la merde »). Le juron, adressé dans un effet réflexif du locuteur à lui-même, s'appuie sur la fonction expressive du langage et permet de ponctuer le discours pour exprimer une émotion («merde!»). L'insulte vise l'interlocuteur dans une fonction impressive, « je te dis merde » ou même « tu es une merde », « espèce de merde ».

Ainsi l'insulte est avant tout un acte de langage interlocutif et porte en elle une force émotionnelle, voire pulsionnelle qui vise l'autre dans la volonté de le rabaisser. Elle joue un rôle éminemment perlocutoire («Parce que je te traite de gros lard, tu vas te sentir comme ça »). Ce fonctionnement-là est rendu possible par des effets linguistiques particuliers (Kerbrat-Orecchioni 1997 : 79). La valeur lexicale des lexèmes joue un rôle important. Il semble évident que certains axiologiques négatifs peuvent être réactivés pour faire injure ${ }^{5}$ : «gros » sera aujourd'hui plus activé que «mince » par exemple. La forme syntaxique, telle la fonction vocative avec des modalités de catégorisation comme « espèce de », accentue aussi la péjoration. Enfin l'intonation est centrale dans l'effet d'injure. Tout terme, même neutre peut se charger d'insulte par la simple force d'évocation, façon de passer du simple constat à l'énoncé injurieux.

Mais, élément fondamental, pour que l'insulte fonctionne pleinement, encore faut-il que l'interlocuteur la perçoive comme telle, en bref qu'elle touche, qu'elle déstabilise et non qu'elle conforte l'autre dans ses croyances. L'insulte existe quand on se sent insulté (Laforest et Moïse 2010).

Distinguer alors les phénomènes linguistiques, les contextes et les intentions des interlocuteurs permet de repérer au mieux les actes de langage qui peuvent être menaçants pour soi et d'éviter, autant que possible, les malentendus conversationnels.

\section{Se repérer dans les usages transgressifs adolescents}

Les adolescents manient avec dextérité ces différents usages pragmatiques, du gros mot à l'insulte. Ils en jouent, provoquent aussi et cherchent même à duper les adultes. Dans la complexité des émotions et sentiments ressentis face aux parlers adolescents, colère,

\footnotetext{
${ }^{5}$ La distinction entre « injure » et « insulte » est peu opérante en linguistique contrairement au domaine juridique. Pour plus de précisions voir Moïse 2008
} 
impuissance, découragement, il semble important de tenter de se repérer pour tout simplement mieux entrer en conversation.

\section{Des gros mots aux ponctuants désémantisés}

Les mots et les pratiques langagières évoluent et les codes langagiers, comme les normes sociales, sont remis en question (quels dont les nouveaux codes de politesse, d'adresse ou de vouvoiement?), parfois avec quelque humeur, à travers des confrontations idéologiques ou générationnelles. Si la dynamique langagière prête souvent à sourire et fait le jeu de bien des ouvrages sur la langue française, elle devient plus sensible et sujet à frottement voire friction quand elle porte sur les mots grossiers ou les jurons. Les mots entrent et sortent de nos besaces lexicales, « les souliers » ne sont plus que « des chaussures », «la voiture » a remplacé «l'auto »; les mots changent de sens, «il craint» ou «il déchire » intransitifs ont lâché leurs compléments ; les mots perdent selon leur temps de leur force sémantique, «collaborer » est aujourd'hui restreint à des partenariats professionnels, sous peine d'évocation historique. Ces changements sont partie prenante des mots courants, mais il en va de même des gros mots, jurons ou mot d'adresse qui fonctionnent de la même façon ; «ma vieille » est redevenue «ma daronne »; «bâtard 6 » a perdu sa force sémantique pour se transformer en mot d'adresse bien souvent affectif (Léglise 2004) et certains enseignants sont choqués par une interpellation comme «salope » à l'égard des filles, mot sans doute en voie de désémantisation (ce qui ne lui retire pas sa valeur de transgression mais la charge est moindre) pour les plus jeunes, à l'image du «con » banalisé dans la langue française. À ce propos, les mots tabou désémantisés font office aussi de ponctuants du discours. «Sa mère » est utilisé aussi comme ponctuant au même titre que le «con » ou le «putain » toulousains, "c'est beau sa mère !" «Ça me prend la tête sa mère »! (Caubet 2001). Mais il faut savoir que ces mots peuvent toujours être réactivés dans les interactions pour servir une insulte et agresser, « $t(u)$ es qu'une salope! » Reste toujours à se replacer en contexte et être au plus des intentions et des interprétations possibles.

\footnotetext{
${ }^{6}$ Les mots tabou sont toutefois le reflet de notre société, des déviances que l'on pointe et stigmatise trop facilement. Si le «bâtard» portait en lui une forme de déviance sociale il n'y pas encore si longtemps, « l'autiste » et « le dépressif » (« espèce de dépressif » peut-on entendre dans les cours de récréation) l'ont remplacé, ce qui peut nous amener aussi à penser la société que nous proposons à nos enfants.
} 
Les jeux expressifs et les vannes rituelles

L'adolescence (voire l'enfance) se construit dans le franchissement des limites et des interdits, entre délectation et expressivité. L'adolescent manie le langage souvent avec brio, faisant peu de cas des modalisateurs («il se peut que», «il faudrait que», «je pense que ») et donc des formes ritualisées de politesse, mais affirmant l'intensité narrative dans ses prises de parole («franchement », "genre », "voilà », « en fait », etc...) ou dans l'adresse à l'autre («t'inquiète», "j'avoue», «t'es pas sérieux!»). Les enfants et adolescents se reconnaissent alors dans leur parler, signe d'entre soi, entre identification, jeu et connivence et emphases, significatifs de leur comportement global à cet âge-là. Dans cette optique, ils ont bien souvent conscience du franchissement des limites, ils savent la plupart du temps s'accommoder aux différents registres attendus (notamment celui de l'école et des adultes). Ils sont plurilingues dans leur langue.

Les vannes se situent donc en marge des injures et font partie de ce jeu entre soi expressif et ludique, parce qu'elles reposent sur une connivence partagée, un jeu quasiment rhétorique, des rituels établis, des codes de solidarité (Lagorgette et Larrivée 2004). Mais c'est un jeu à risque, et, quand elles sont perçues comme blessantes, les vannes peuvent basculer dans la provocation, particulièrement si les deux protagonistes ne participent pas au jeu comme ce peut être le cas entre un élève et une enseignante. Dans une situation de classe (Moïse 2006), un élève repris maintes fois par une enseignante osera la tancer par un «vous voulez que je parte plus vous donnez un avertissement plus des devoirs plus des rédactions mais : + vous voulez pas de l'or en plus » qui la laissera sans voix. S'il permet à l'élève de sortir vainqueur de l'interaction aux yeux de ses pairs, le jeu de la grillade non partagé en situation dissymétrique ne peut qu'engendrer réprimande et sanction et enfermé alors l'adolescent sans une identité négative assignée.

\section{L'insulte comme dernier recours et une transgression affirmée en guise de souffrance}

Au-delà des pratiques ludiques, des joutes verbales et des stratégies décalées ${ }^{7}$, la violence verbale, qu'elle se manifeste contre soi par le juron, ou contre l'autre par la

\footnotetext{
${ }^{7}$ Il est nécessaire pour les adultes de savoir repérer (ce qui est souvent difficile) les limites ludiques des pratiques adolescentes. On joue disent-ils bien souvent. Peut-être, mais qui joue? Tous les jeunes en train de discuter, ou certains au détriment d'autres ? Et à quel moment un terme sera-t-il blessant parce que recouvrant tout son sens (l'insulte à la mère, l'insulte aux morts) ? Quand essaie-t-on « d'embrouiller » un adulte ? Prise en compte du contexte et intervention d'un tiers sont alors souvent indispensables pour dénouer certains malentendus et incompréhensions.
} 
provocation, la menace ou l'insulte, dit toujours quelque chose. Tout est sans doute une question de degré. Ceux qui sont considérés comme les plus irrespectueux sont ceux qui demandent le plus de respect. Pour le dire autrement, l'irrespect serait alors une demande forte de respect, c'est-à-dire une demande de reconnaissance et de sécurisation quand l'estime de soi vacille ${ }^{8}$. Comme j'aime à le dire, la violence verbale est un symptôme de mal être; elle est à lier à d'autres pratiques langagières et psychologiques comme la victimisation ou la prise de pouvoir. Elle rend compte alors de sentiment d'injustice (vérifiable ou pas), de non reconnaissance ou de frustration (désir inassouvi). Tout le travail consiste en un décentrement, en une compréhension de ses propres besoins et manques et en une valorisation de soi. Ainsi toute forme d'agressivité ${ }^{9}$, si elle doit être refusée et sanctionnée, doit être aussi revisitée, parlée, comprise par un tiers. La rémédiation ne pourra pas non plus seulement reposer sur le langage mais aussi sur la prise en compte de l'enfant ou l'adolescent dans sa globalité, avec ses désirs et ses empêchements. On pourra alors comprendre que la violence verbale est une façon efficace de se donner à voir, quand on veut échapper à soi-même, s'affirmer face à l'autre, parce que le sujet adolescent en construction (et particulièrement s'il est blessé) sait plus ce qu'il n'est pas que ce qu'il est.

Je voudrais appuyer ici ma réflexion sur le suivi d'adolescents auteur et/ou victime de violences sexuelles ${ }^{10}$ (Gamet et Moïse 2010). Les troubles du développement de la sexualité s'accompagnent parfois chez l'enfant, notamment s'il a subi d'autres formes de maltraitance, de difficultés relationnelles et sociales, de comportements physiques et verbaux empreints d'agressivité. Ainsi, de telles attitudes, qui peuvent laisser perplexes l'entourage ou les professionnels, sont bien souvent le symptôme de souffrances non révélées. La violence, qu'elle soit physique ou verbale, auto- ou hétéro-dirigée, quand elle dépasse l'impulsivité caractéristique de l'enfance et de l'adolescence ${ }^{11}$ dont je viens de parler, est significative d'un mal-être intime et profond.

\footnotetext{
${ }^{8}$ On sait que les manques d'assurance et les fragilités dans la construction de soi reposent sur des ruptures dans l'attachement lors de la petite enfance.

${ }^{9}$ L'agressivité (du latin adgredior, aller vers, se rapprocher) est un franchissement du territoire de l'autre (physique et symbolique) mais aussi une tentative de rapprochement et de contact.

10 Il s'agit ici de suivis de thérapie sexuelle menés par Marie-Laure Gamet. J'ai donc eu accès à certains cas cliniques qui sont analysés dans notre ouvrage (Gamet et Moïse 2010).

11 Au-delà de l'impulsivité adolescente, certains font le choix (Berger 2008 : 226), chez les enfants et adolescents, de ce qui relève d'un côté de la violence «extrême » engendrée par des environnements familiaux destructurants et de l'autre de la violence « impulsive » liée à une « dimension de tempérament constitutionnel ». Pour ma part, je considère qu'il faut faire le pari, dans le temps de l'enfance et de
} 
Les signes de violence caractérisée chez les enfants ne naissent pas ex nihilo. Il s'agit ici de considérer les comportements violents des enfants et adolescents en ce qu'ils sont conditionnés par un environnement familial « défectueux » et "désastreux » dès leur plus jeune âge, notamment, au-delà d'une certaine insécurisation, à cause de « l'exposition à la violence et à l'échec de la séduction primaire » (Berger $2008: 48$ ) et d'une absence d'attachement (Gamet et Moïse 2010). Ces enfants sont non seulement confrontés à la violence mais aussi jugés non attirants par leurs parents et donc physiquement délaissés. Quand on n'est pas dans l'inhibition, les violences manifestées s'expriment le plus souvent à travers des comportements destructeurs et incontrôlés, physiques et verbaux.

Je voudrais pour illustrer mon propose citer les rapports éducatifs de Bertrand placé dans des maisons d'enfants, rapports éloquents qui rendent compte des excès de violence notamment verbale de ce jeune adolescent et donc de sa difficulté à s'intégrer socialement.

9 août 2004. Grossier et insultant à l'égard des jeunes et des animateurs. Actes violents. A déshabillé un garçon. 3 décembre 2004. Etablissement d'enseignement adapté. Refus de tout et indifférence. Septembre-novembre 2004. Attitudes à l'internat. Insulte et menace un enseignant: « Ta gueule putain. Tu vas voir ta gueule vendredi, ma mère va te péter la gueule ». / Insulte et menace un enseignant : "Ma mère va venir te buter, ta mère, la pute... ». / Très insolent. / Giffle, bouscule, frappe, tape, lance. / Insolent et irrespectueux envers l'adulte. /Violence verbale et physique. Insulte et tutoie les enseignants. / Insolent, grossier : « me pète pas les couilles. Je vais t'en coller une. Fais pas la maline ». Insulte, menace : «va te faire foutre, nique ta mère, enculée » « je vais aller chercher mon frère, tu vas voir ».

25 août 2005. Ses objectifs étaient de continuer à respecter le cadre, les adultes et les autres jeunes, de s'efforcer à contrôler son langage. / Les derniers événements montrent que Bertrand n'arrive, ni à se contenir, ni à canaliser son agressivité. / Il est dans le déni. Les sanctions et les plaintes successives ne semblent pas l'aider à réfléchir. / Il a du mal à verbaliser, il est sans doute en position d'évitement verbal, de non verbalisation, de mise à distance de la parole et de ses contenus. / On ne peut cependant évoquer d'éléments pervers.

22 décembre 2006. Suite à des insultes d'un camarade, claque et « on va te couper la bite à coup de serpette $»$.

15 février 2007. Comportement agressif, vulgaire et non coopératif.

25 avril 2007. Repris quotidiennement pour son vocabulaire vulgaire, très souvent à connotation sexuelle. Une semaine d'exclusion pour agression verbale. Il est sanctionné. Invectives verbales incessantes. Irrespect à l'égard de ses camarades et des adultes. Vocifère.

La violence verbale telle que décrite dans ces rapports permet d'expliciter, au-delà d'une impossibilité à se dire et à se penser, des processus de défense. Il est inepte de croire

l'adolescence, d'inflexions et de changements subjectifs possibles comme le montrent les prises en charge thérapeutiques. 
que l'enfant n'a ni les mots ni les arguments à sa disposition, il les a à partir du moment où on lui donne la possibilité d'une réflexion sur soi, entre fermeté et écoute. Pour le dire autrement, ce n'est pas une question de «pauvreté lexicale et argumentative » comme on peut l'entendre parfois, mais de difficultés psychiques pour se regarder et identifier ses émotions qui sont à l’œuvre.

Regardons de plus près ce qui est dit dans ces rapports. La violence verbale est ici fulgurante, marquée par l'insulte et la menace, actes de langage directs, elle n'est pas la manifestation de comportements pervers ou manipulateurs. L'agressivité exprimée est le signe de défense de territoires, pulsion réactive qui vise à préserver une certaine intégrité physique quand toute tentative de rapprochement est ressentie comme une attaque menaçante. L'agressivité, sous forme de défense, conforte alors l'impossibilité à se verbaliser et entretient une pensée dogmatique rassurante faite de certitudes, de généralisation, d'émotions projetées sur le monde extérieur ( « Il a du mal à verbaliser, il est sans doute en position d'évitement verbal, de non verbalisation, de mise à distance de la parole et de ses contenus »). Dans une projection d'un acte physique, la menace qui accompagne la violence fulgurante permet de maintenir un fantasme de protection impossible (« Ma mère va venir te buter / je vais aller chercher mon frère »). L'insulte enfin comme dernier recours veut signifier, par un effet d'essentialisation, la négation de l'autre, façon de projeter son propre sentiment de manque, de vide et de mésestime. Dévaloriser l'autre permet de se valoriser soi-même. Sans oublier que menace et insulte sont chez Bertrand, plus qu'à l'ordinaire, à contenu hautement sexuel, marque d'un malêtre sexuel («On va te couper la bite à coup de serpette / pointeur»). Ecouter la violence verbale donne alors avant tout à entendre les carences affectives et les troubles du comportement chez ces adolescents violentés, facteurs d'inadaptation sociale et scolaire.

\section{Conclusion}

Notre société contemporaine a certes parfois du mal à supporter des comportements adolescents provocateurs jugés «violents», bagarres, incivilités notamment, qui seraient passés inaperçus il y a quelques années (Neyrand (Ed.) 2006) mais je voudrais pour ma part insister sur la complexité des pratiques langagières actuelles. Les codes de politesse sont mouvants, les usages pragmatiques sujets à malentendus (entre ponctuant désémantisé et insulte de souffrance la distinction est indispensable) et les tensions générationnelles à vif. Ainsi, s'il s'agit parfois de regarder avec tolérance 
certains changements linguistiques, significatifs de l'évolution des normes sociales et loin d'être nouveaux, il ne faut pas pour autant sous-estimer des attitudes qui s'avèrent constitutives et pathologiques de certaines personnalités et traduisent un mal-être profond. Tout est alors question de formation, d'écoute et d'empathie.

\section{Références bibliographiques}

Berger M., 2008, «Voulons-nous des enfants barbares. Prévenir et traiter la violence extrême. », Paris, Dunod

Caubet D., 2001, « Du baba (papa) à la mère, des emplois parallèles en arabe marocain et dans les parlures jeunes en France », in Les langues déliées, CAHIERS D’ETUDES AFriCAINES numéro 163-164: 735-748

Delvau A., [1900], 1990, Dictionnaire érotique moderne, Paris, Les Editions

Duchêne A. et Moïse C., 2010, «Genre et sexualité : quels enjeux pour les sciences du langage ?», Duchêne A. et Moïse C. (Eds), Langage, genre et sexualité, Montréal, Éditions Nota bene : 7-26

Gamet M-L. et Moïse C., 2010, 2010 La violence sexuelle des mineurs, auteurs et victimes. De la parole aux soins, Paris, Dunod

Huston N., 2002, Dire et interdire, Paris, Payot

Kerbrat-Orecchioni C., 1997, L'énonciation, Paris, Armand Colin

Laforest M. et Moïse C., 2010, « Entre reproche et insulte, comment définir les actes de condamnation », communication aux journées De la satire à la violence verbale : quand l'humour-l'humeur engendre l'affrontement, 25-26 novembre, MSH, Paris 8.

Lagorgette D. et Larrivée P., 2004, «Interprétation des insultes et relations de solidarité », Lagorgette D. et Larrivée P. (2004) (Eds.), Les insultes : approches sémantiques et pragmatiques, LANGUE FRANÇAISE 144, Larousse : 83-103

Léglise I., 2004, «Diversité des formes d'oral et rapport au langage chez les travailleurs sociaux : l'exemple des médiateurs urbains», Léglise I. (Ed.), Pratiques, langues et discours dans le travail social, Paris, L'Harmattan : 29-52

Moïse C., 2006, « Analyse de la violence verbale : quelques principes méthodologiques », in actes des XXVIe Journées d'étude sur la parole, 12-16 juin, Dinard, http://jep2006.irisa.fr/JEP06 ACTES.PDF 
Moise C., 2008, «Formes et valeurs de l'injure dans les processus de construction identitaire », Tauzin A. (Éd), Insultes, injures et vannes en France et au Maghreb, Paris, Kathala : 175-196

Moïse C. et Schultz-Romain C., à paraître, Violence verbale et listes de discussions : les argumentations polémiques », CAHIERS DE L'INSTITUT DE LINGUISTIQUE DE LOUVAIN

Neyrand G. (Ed.), 2006, Faut-il avoir peur de nos enfants? Politiques sécuritaires et enfance, Paris, La découverte

Rouayreng C., 1996, Les gros mots, Paris, Que sais-je ? Puf

Vincent D., 1984, « Le sacre au Québec : transgression d'un ordre religieux ou social ?», CULTuRE, vol.4, numéro 2 : 55-61 